\title{
Triphenylphosphite as a good reagent for the diasteroselective synthesis of phosphonate esters
}

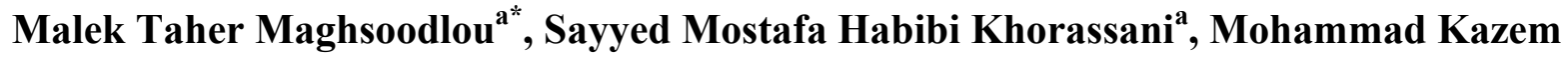 \\ Rofouei $^{\mathrm{b}}$, Sayyed Reza Adhamdoust, ${ }^{\text {a }}$ and Mahmoud Nassiri ${ }^{\mathrm{a}}$ \\ a Department of Chemistry, The University of Sistan and Balouchestan, P. O. Box 98135-674, \\ Zahedan, Iran \\ b Faculty of Chemistry, The University of Tarbiat Moallem, Tehran, Iran \\ E-mail: MTmaghsoodlou@yahoo.com
}

\begin{abstract}
The reaction between dialkyl acetylenedicarboxylates and $\mathrm{N}-\mathrm{H}$ acids such as pyrazole and indazole in the presence of triphenylphosphite at room temperature led to stable phosphonate ester derivatives $\mathbf{4 a - f}$. The configuration of compounds $\mathbf{4 a - f}\left(\mathbf{2} \mathbf{S}^{*}, \mathbf{3} \mathbf{R}^{*}\right)$ was determined on the basis of coupling constants predicted from the Karplus equation.
\end{abstract}

Keywords: N-H acids, acetylenic esters, triphenylphosphite, Karplus equation, diastereomer, phosphonate esters

\section{Introduction}

In recent years there has been an increasing interest in the synthesis of organophosphorus compounds, in particular those bearing a carbon atom bound to a phosphorus atom. This interest has resulted from the recognition of the value of such compounds in a wide range of industrial, biological and chemical synthetic aspects. ${ }^{1-4,20-22}$ The successful attack by nucleophilic trivalent phosphorus on a carbon atom is facilitated when the latter is part of, or conjugated with, a carbonyl group, or when it is part of an unsaturated bond otherwise activated. ${ }^{2-10}$ There are many studies on the reaction between trivalent phosphorus nucleophiles and $\alpha, \beta$-unsaturated carbonyl compounds in the presence of a proton source such as an alcohol or a phenol., ${ }^{2,10,11}$ New or improved methods for phosphonate synthesis continue to attract much attention because phosphonates have biologically important properties and serve as natural products, analogues of phosphates, phosphonopeptides, amino acid analogues and pro drugs. Also the phosphonate esters have physiological activity within the cell.$^{12-14,17-19}$ 

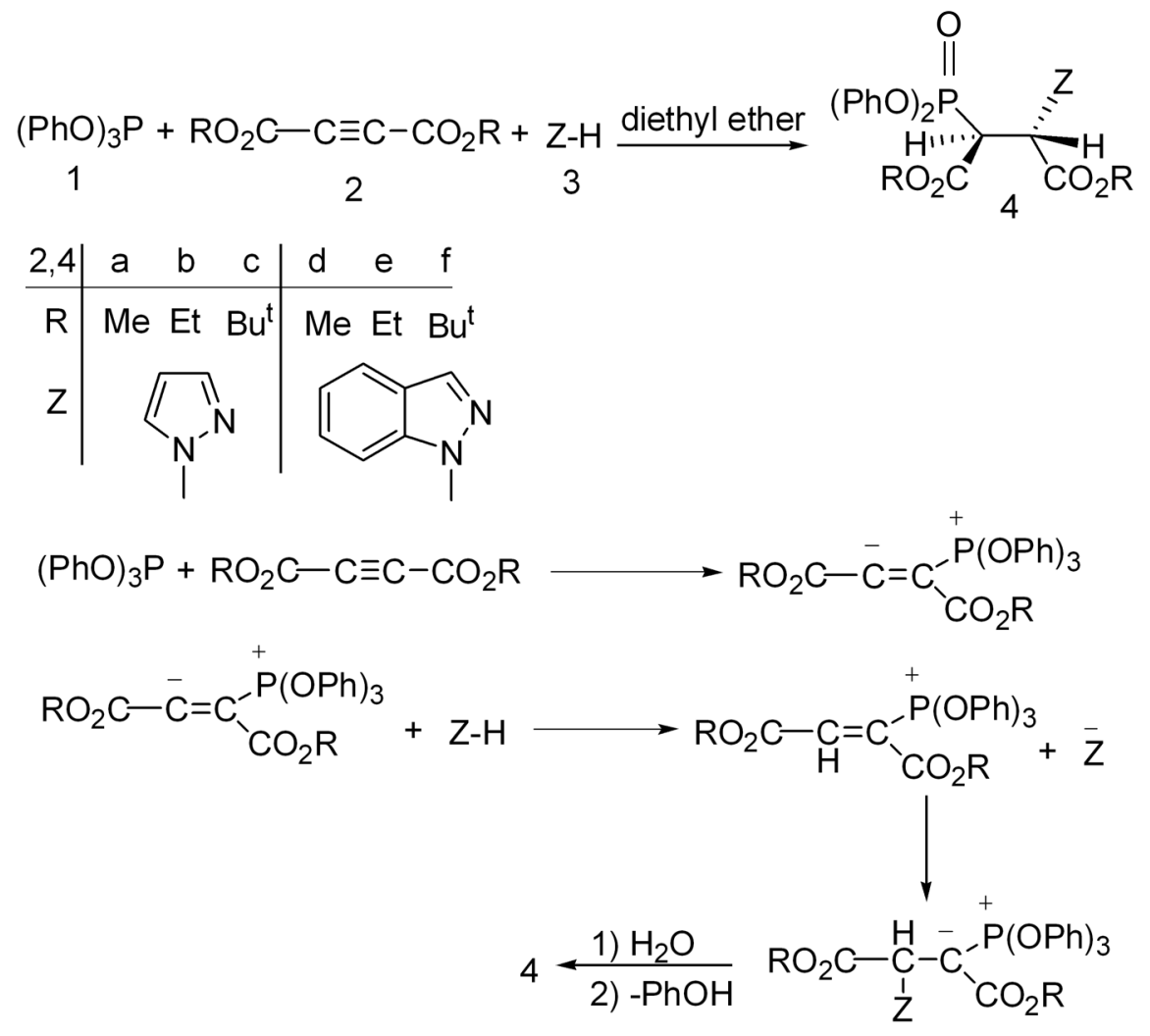

\section{Scheme 1}

\section{Results and Discussion}

In the current work, we wish to report a simple, short, neutral stereoselective synthesis of phosphonate esters at room temperature from reaction between triphenylphosphite $\mathbf{1}$ and acetylenic esters $\mathbf{2}$ in the presence of N-H acids. The use of pyrazole and indazole $\mathbf{3}$ led to $\mathbf{4}$ in fairly high yield (see Scheme 1). These reactions were carried out in diethyl ether as solvent at room temperature and were finished within a few hours. The ${ }^{1} \mathrm{H}$ and ${ }^{13} \mathrm{C}$ NMR spectrum of the crude product clearly indicated the formation of phosphonate esters 4a-f. Any products other than 4a-f could not be detected by NMR spectroscopy. The structures of compounds 4a-f were deduced from their IR, ${ }^{1} \mathrm{H},{ }^{13} \mathrm{C}$ and ${ }^{31} \mathrm{P}$ NMR spectra. The $500 \mathrm{MHz}{ }^{1} \mathrm{H}$ NMR spectra of compound 4a displayed two sharp lines $(\delta=3.71,3.79)$ arising from methoxy protons, along with signals for methine protons at $\delta=4.62 \mathrm{ppm}\left({ }^{2} \mathrm{~J}_{\mathrm{PH}}=21.2 \mathrm{~Hz},{ }^{3} \mathrm{~J}_{\mathrm{HH}}=10.8 \mathrm{~Hz}\right)$ and $\delta=5.82 \mathrm{ppm}$ $\left({ }^{3} \mathrm{~J}_{\mathrm{PH}}=8.4 \mathrm{~Hz},{ }^{3} \mathrm{~J}_{\mathrm{HH}}=10.8 \mathrm{~Hz}\right)$ which appear as two doublets of doublet, respectively, for the $\mathrm{O}=\mathrm{P}-$ $\mathrm{CH}-\mathrm{CH}$ and $\mathrm{O}=\mathrm{P}-\mathrm{CH}-\mathrm{CH}$ groups. The vicinal proton-proton coupling constant $\left({ }^{3} \mathrm{~J}_{\mathrm{HH}}\right)$ as a function of the torsion angle can be obtained from the Karplus equation. ${ }^{1,15}$ Typically, $J_{\text {gauche }}$ varies between 1.5 and $5 \mathrm{~Hz}$ and $J_{\text {anti }}$ between 10 and $14 \mathrm{~Hz}$. Observation of ${ }^{3} J_{\mathrm{HH}}=10.8 \mathrm{~Hz}$ for the vicinal protons in compound 4a (see Experimental section) indicates an anti arrangement for 
these protons. Since compound 4a possess two stereogenic centers, two diastereoisomers with anti $\mathrm{HCCH}$ arrangements are possible. The three-bond carbon-phosphorus coupling, ${ }^{3} J_{\mathrm{CP}}$, depends on the configuration, as expected, the transoid coupling being larger than the cisoid ones. The Karplus relation can be derived from the data for organophosphorus compounds with tetra and pentavalent phosphorus. ${ }^{16}$ The observation of ${ }^{3} J_{\mathrm{CP}}$ of $18.6 \mathrm{~Hz}$ for the ester $\mathrm{C}=\mathrm{O}$ group (see Experimental section), is in a good agreement with the $2 S^{*}, 3 R^{*}-4 a$ and its mirror image $2 \mathrm{R}^{*}, 3 \mathrm{~S}^{*}-4 \mathbf{a}$ geometries (see Scheme 2). Although the presence of the ${ }^{31} \mathrm{P}$ nucleus complicates both the ${ }^{1} \mathrm{H}$ and ${ }^{13} \mathrm{C}$ NMR spectra of $\mathbf{4 a}$, it helps in the assignment of the signals by long-range couplings with the ${ }^{1} \mathrm{H}$ and ${ }^{13} \mathrm{C}$ nuclei (see Experimental section). The ${ }^{1} \mathrm{H}$ and ${ }^{13} \mathrm{C}$ NMR spectra of (4b-f) are similar to those of $\mathbf{4 a}$, except for the ester groups, which exhibited characteristic resonances with appropriate chemical shifts (see Experimental section). The structural assignments made on the basis of the ${ }^{1} \mathrm{H}$ and ${ }^{13} \mathrm{C}$ NMR spectra of compounds (4a-f) were supported by the IR spectra. The carbonyl region of the spectra exhibited two distinct absorption bands for each compound (see Experimental section). Of special interest is the ester absorption at $1745-1719 \mathrm{~cm}^{-1}$ for these compounds.
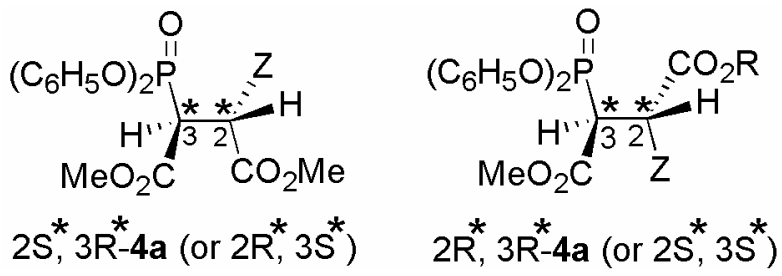

Scheme 2

\section{Experimental Section}

General Procedures. Melting points and IR spectra of all compounds were measured on an Electrothermal 9100 apparatus and a Shimadzu IR-460 spectrometer respectively. Also, the ${ }^{1} \mathrm{H}$, ${ }^{13} \mathrm{C}$, and ${ }^{31} \mathrm{P}$ NMR spectra were obtained from a BRUKER DRX-500 AVANCE instrument with $\mathrm{CDCl}_{3}$ as solvent at $500.1,125.8$, and $202.4 \mathrm{MHz}$ respectively. In addition, the mass spectra were recorded on a Shimadzu QP 1100 EX mass spectrometer operating at an ionization potential of $70 \mathrm{eV}$. Elemental analysis for $\mathrm{C}, \mathrm{H}$ and $\mathrm{N}$ were performed using a Heraeus CHN-ORapid analyzer. Dialkyl acetylenedicarboxylates, triphenlphosphite, pyrazole and indazole were purchased from Fluka, (Buchs, Switzerland) and used without further purifications.

Preparation of $\left(2 S, 3 R^{*}\right)$-dimethyl-2-(pyrazole-1-yl)-3-(diphenoxyphosphonato)butane dioate (4a). To a magnetically stirred solution of triphenylphosphite $(0.31 \mathrm{~g}, 1 \mathrm{mmol})$ and pyrazole $(0.07 \mathrm{~g}, 1 \mathrm{mmol})$ in diethyl ether $(10 \mathrm{ml})$ was added dropwise a mixture of dimethyl acetylenedicarboxylate $(0.14 \mathrm{~g}, 1 \mathrm{mmol})$ in diethyl ether $(5 \mathrm{ml})$ at $-10{ }^{\circ} \mathrm{C}$ over $10 \mathrm{~min}$. After 
approximately 24 hours stirring at room temperature, the solvent was removed under reduced pressure and product washed with cold diethyl ether $(2 \times 5 \mathrm{~mL})$.

White powder, 97\%, mp=125-127 ${ }^{\circ} \mathrm{C}$. IR $(\mathrm{KBr})\left(v_{\max }, \mathrm{cm}^{-1}\right)$ : 1745 and $1719(\mathrm{C}=\mathrm{O}), 1271(\mathrm{P}=\mathrm{O})$. Anal. Calcd for $\mathrm{C}_{21} \mathrm{H}_{21} \mathrm{~N}_{2} \mathrm{O}_{7} \mathrm{P}$ (444): C, 56.63; H, 4.80; N, 6.25, Found: C, 56.76; H, 4.73; N, 6.31. ${ }^{1} \mathrm{H}$ NMR (500.1 MHz, $\left.\delta, \mathrm{CDCl}_{3}\right): 3.71$ and $3.79\left(6 \mathrm{H}, 2 \mathrm{~s}, 2 \mathrm{CH}_{3}\right), 4.62\left(1 \mathrm{H}, \mathrm{dd},{ }^{2} \mathrm{~J}_{\mathrm{PH}}=21.2\right.$ $\left.\mathrm{Hz},{ }^{3} \mathrm{~J}_{\mathrm{HH}}=10.8 \mathrm{~Hz}, \mathrm{P}-\mathrm{CH}-\mathrm{CH}\right), 5.82\left(1 \mathrm{H}, \mathrm{dd},{ }^{3} \mathrm{~J}_{\mathrm{PH}}=8.4 \mathrm{~Hz},{ }^{3} \mathrm{~J}_{\mathrm{HH}}=10.8 \mathrm{~Hz}, \mathrm{P}-\mathrm{CH}-\mathrm{CH}\right), 6.28-7.66$ $\left(13 \mathrm{H}, \mathrm{m}, \mathrm{H}_{\text {aro }}\right) .{ }^{13} \mathrm{C} \mathrm{NMR}\left(125.8 \mathrm{MHz}, \delta, \mathrm{CDCl}_{3}\right): 47.96\left(\mathrm{~d},{ }^{1} \mathrm{~J}_{\mathrm{CP}}=134.8 \mathrm{~Hz}, \mathrm{P}-\mathrm{CH}\right), 53.05$ and $53.20\left(\mathrm{~s}, 2 \mathrm{OCH}_{3}\right), 61.72\left(\mathrm{~d},{ }^{2} \mathrm{~J}_{\mathrm{CP}}=3.5 \mathrm{~Hz}, \mathrm{P}-\mathrm{C}-\mathrm{CH}\right), 106.20\left(1 \mathrm{C}, \mathrm{C}_{3} \mathrm{H}_{3} \mathrm{~N}_{2}\right), 120.04120 .30(2 \mathrm{~d}$, ${ }^{3} \mathrm{~J}_{\mathrm{PC}}=4.7 \mathrm{~Hz} \mathrm{C}_{\text {ortho }}$ of $\left.2 \mathrm{C}_{6} \mathrm{H}_{5}\right), 125.29$ and $125.39\left(\mathrm{C}_{\text {para }}\right.$ of $\left.2 \mathrm{C}_{6} \mathrm{H}_{5}\right), 129.64$ and $129.73\left(\mathrm{C}_{\text {meta }}\right.$ of $\left.2 \mathrm{C}_{6} \mathrm{H}_{5}\right), 131.92$ and $141.17\left(2 \mathrm{C}, \mathrm{C}_{3} \mathrm{H}_{3} \mathrm{~N}_{2}\right), 149.47$ and $149.88\left(2 \mathrm{~d},{ }^{2} \mathrm{~J}_{\mathrm{CP}}=9.0 \mathrm{~Hz}, \mathrm{C}_{\text {ipso }}\right.$ of $\left.2 \mathrm{C}_{6} \mathrm{H}_{5}\right)$, $166.64\left(\mathrm{~d},{ }^{3} \mathrm{~J}_{\mathrm{CP}}=6.2 \mathrm{~Hz}, \mathrm{C}=\mathrm{O}\right), 167.04\left(\mathrm{~d},{ }^{2} \mathrm{~J}_{\mathrm{CP}}=18.6 \mathrm{~Hz}, \mathrm{C}=\mathrm{O}\right) .{ }^{31} \mathrm{P}$ NMR $(202.4 \mathrm{MHz}, \delta, 10.13$ $\left[\mathrm{s},(\mathrm{PhO})_{2} \mathrm{P}(=\mathrm{O})\right]$.

$\left(2 S^{*}, 3 R^{*}\right)$-Diethyl-2-(pyrazole-1-yl)-3-(diphenoxyphosphonato)butanedioate $(4 \mathrm{~b})$. White powder, 96\%, mp=124-126 ${ }^{\circ} \mathrm{C}$. IR $(\mathrm{KBr})\left(v_{\max }, \mathrm{cm}^{-1}\right): 1741$ and $1719(\mathrm{C}=\mathrm{O}), 1269(\mathrm{P}=\mathrm{O})$. Anal. Calcd for $\mathrm{C}_{23} \mathrm{H}_{25} \mathrm{~N}_{2} \mathrm{O}_{7} \mathrm{P}$ (472): C, 58.35; H, 5.35; N, 6.02, Found: C, 58.47; H, 5.30; N, 5.93. ${ }^{1} \mathrm{H}$ $\operatorname{NMR}\left(500.1 \mathrm{MHz}, \delta, \mathrm{CDCl}_{3}\right): 1.14$ and $1.25\left(6 \mathrm{H}, 2 \mathrm{t},{ }^{3} \mathrm{~J}_{\mathrm{HH}}=7.1 \mathrm{~Hz}, 2 \mathrm{CH}_{3}\right), 4.16$ and $4.25(4 \mathrm{H}, \mathrm{m}$, $2 \mathrm{ABX}_{3}$ system, 2OCH 2$), 4.60\left(1 \mathrm{H}, \mathrm{dd},{ }^{2} \mathrm{~J}_{\mathrm{PH}}=21.2 \mathrm{~Hz},{ }^{3} \mathrm{~J}_{\mathrm{HH}}=11.0 \mathrm{~Hz}, \mathrm{P}-\mathrm{CH}-\mathrm{CH}\right), 5.77(1 \mathrm{H}$, dd, $\left.{ }^{3} \mathrm{~J}_{\mathrm{PH}}=8.8 \mathrm{~Hz},{ }^{3} \mathrm{~J}_{\mathrm{HH}}=11.0 \mathrm{~Hz}, \mathrm{P}-\mathrm{CH}-\mathrm{CH}\right), 6.24-7.64\left(13 \mathrm{H}, \mathrm{m}, \mathrm{H}_{\mathrm{aro}}\right) .{ }^{13} \mathrm{C} \mathrm{NMR}(125.8 \mathrm{MHz}, \delta$, $\left.\mathrm{CDCl}_{3}\right)$ : 13.77 and 13.82(2CH$), 48.06\left(\mathrm{~d},{ }^{1} \mathrm{~J}_{\mathrm{CP}}=134.6 \mathrm{~Hz}, \mathrm{P}-\mathrm{CH}\right), 62.01\left(\mathrm{~d},{ }^{2} \mathrm{~J}_{\mathrm{CP}}=3.3 \mathrm{~Hz}, \mathrm{P}-\mathrm{C}-\right.$ $\mathrm{CH}), 62.41$ and $62.55\left(2 \mathrm{OCH}_{2}\right), 106.07\left(1 \mathrm{C}, \mathrm{C}_{3} \mathrm{H}_{3} \mathrm{~N}_{2}\right), 120.24$ and $120.44\left(2 \mathrm{~d},{ }^{3} \mathrm{~J}_{\mathrm{PC}}=4.6 \mathrm{~Hz}\right.$ $\mathrm{C}_{\text {ortho }}$ of $\left.2 \mathrm{C}_{6} \mathrm{H}_{5}\right), 125.39$ and $125.45\left(\mathrm{C}_{\text {para }}\right.$ of $\left.2 \mathrm{C}_{6} \mathrm{H}_{5}\right), 129.64$ and $129.66\left(\mathrm{C}_{\text {meta }}\right.$ of $\left.2 \mathrm{C}_{6} \mathrm{H}_{5}\right)$, 131.97 and $141.22\left(2 \mathrm{C}, \mathrm{C}_{3} \mathrm{H}_{3} \mathrm{~N}_{2}\right), 149.72$ and $150.07\left(2 \mathrm{~d},{ }^{2} \mathrm{~J}_{\mathrm{CP}}=9.6 \mathrm{~Hz}, \mathrm{C}_{\text {ipso }}\right.$ of $\left.2 \mathrm{C}_{6} \mathrm{H}_{5}\right), 166.22$ $\left(\mathrm{d},{ }^{3} \mathrm{~J}_{\mathrm{CP}}=6.0 \mathrm{~Hz}, \mathrm{C}=\mathrm{O}\right), 167.08\left(\mathrm{~d},{ }^{2} \mathrm{~J}_{\mathrm{CP}}=19.0 \mathrm{~Hz}, \mathrm{C}=\mathrm{O}\right) .{ }^{31} \mathrm{P}$ NMR $(202.4 \mathrm{MHz}, \delta, 10.85[\mathrm{~s}$, $\left.(\mathrm{PhO})_{2} \mathrm{P}=\mathrm{O}\right]$.

$\left(2 S^{*}, 3 R^{*}\right)$-Di-tert-butyl -2-(pyrazole-1-yl)-3-(diphenoxyphosphonato)butanedioate (4c). White powder, 92\%, mp=124-126 ${ }^{\circ} \mathrm{C} . . \mathrm{IR}(\mathrm{KBr})\left(v_{\max }, \mathrm{cm}^{-1}\right)$ : 1739 and $1722(\mathrm{C}=\mathrm{O}), 1273(\mathrm{P}=\mathrm{O})$. Anal. Calcd for $\mathrm{C}_{27} \mathrm{H}_{33} \mathrm{~N}_{2} \mathrm{O}_{7} \mathrm{P}$ (528): C, 61.50; H, 6.19; N, 5.37, Found: C, 61.36; H, 6.25; N, 5.30. ${ }^{1} \mathrm{H}$ NMR $\left(500.1 \mathrm{MHz}, \delta, \mathrm{CDCl}_{3}\right): 1.36$ and $1.47(18 \mathrm{H}, 2 \mathrm{CMe}), 4.52\left(1 \mathrm{H}, \mathrm{dd},{ }^{2} \mathrm{~J}_{\mathrm{PH}}=20.9\right.$ $\left.\mathrm{Hz},{ }^{3} \mathrm{~J}_{\mathrm{HH}}=11.1 \mathrm{~Hz}, \mathrm{P}-\mathrm{CH}-\mathrm{CH}\right), 5.64\left(1 \mathrm{H}, \mathrm{dd},{ }^{3} \mathrm{~J}_{\mathrm{PH}}=8.6 \mathrm{~Hz},{ }^{3} \mathrm{~J}_{\mathrm{HH}}=11.1 \mathrm{~Hz}, \mathrm{P}-\mathrm{CH}-\mathrm{CH}\right), 6.24-7.61$ $\left(13 \mathrm{H}, \mathrm{m}, \mathrm{H}_{\mathrm{aro}}\right) .{ }^{13} \mathrm{C} \mathrm{NMR}\left(125.8 \mathrm{MHz}, \delta, \mathrm{CDCl}_{3}\right): 27.62$ and $27.70\left(2 \mathrm{CMe}_{3}\right), 48.98(\mathrm{~d}$, $\left.{ }^{1} \mathrm{~J}_{\mathrm{CP}}=135.7 \mathrm{~Hz}, \mathrm{P}-\mathrm{CH}\right), 62.78\left(\mathrm{~d},{ }^{2} \mathrm{~J}_{\mathrm{CP}}=3.4 \mathrm{~Hz}, \mathrm{P}-\mathrm{C}-\mathrm{CH}\right), 83.33$ and $83.41\left(2 \mathrm{CMe}_{3}\right), 105.97(1 \mathrm{C}$, $\left.\mathrm{C}_{3} \mathrm{H}_{3} \mathrm{~N}_{2}\right), 120.38$ and $120.69\left(2 \mathrm{~d},{ }^{3} \mathrm{~J}_{\mathrm{PC}}=4.5 \mathrm{~Hz} \mathrm{C}_{\text {ortho }}\right.$ of $\left.2 \mathrm{C}_{6} \mathrm{H}_{5}\right), 125.33$ and $125.49\left(\mathrm{C}_{\text {para }}\right.$ of $\left.2 \mathrm{C}_{6} \mathrm{H}_{5}\right), 129.26$ and $129.64\left(\mathrm{C}_{\text {meta }}\right.$ of $\left.2 \mathrm{C}_{6} \mathrm{H}_{5}\right), 131.62$ and $140.86\left(2 \mathrm{C}, \mathrm{C}_{3} \mathrm{H}_{3} \mathrm{~N}_{2}\right), 149.81$ and $149.95\left(2 \mathrm{~d},{ }^{2} \mathrm{~J}_{\mathrm{CP}}=9.4 \mathrm{~Hz}, \mathrm{C}_{\text {ipso }}\right.$ of $\left.2 \mathrm{C}_{6} \mathrm{H}_{5}\right), 163.64\left(\mathrm{~d},{ }^{3} \mathrm{~J}_{\mathrm{CP}}=6.0 \mathrm{~Hz}, \mathrm{C}=\mathrm{O}\right), 165.98\left(\mathrm{~d},{ }^{2} \mathrm{~J}_{\mathrm{CP}}=19.7\right.$ $\mathrm{Hz}, \mathrm{C}=\mathrm{O}) .{ }^{31} \mathrm{P}$ NMR $\left(202.4 \mathrm{MHz}, \delta, 10.85\left[\mathrm{~s},(\mathrm{PhO})_{2} \mathrm{P}=\mathrm{O}\right]\right.$.

$(\mathbf{2} S * \mathbf{3} \boldsymbol{R} *$ )-Dimethyl-2-(indazole-1-yl)-3-(diphenoxyphosphonato)butanedioate $(4 \mathrm{~d}) . \quad$ White powder, 98\%, mp=132-134 ${ }^{\circ} \mathrm{C}$. IR $(\mathrm{KBr})\left(v_{\max }, \mathrm{cm}^{-1}\right): 1745$ and $1719(\mathrm{C}=\mathrm{O}), 1271(\mathrm{P}=\mathrm{O})$. Anal. Calcd for $\mathrm{C}_{25} \mathrm{H}_{23} \mathrm{~N}_{2} \mathrm{O}_{7} \mathrm{P}$ (494): C, 61.03; H, 4.51; N, 5.73, Found: C, 60.73; H, 4.66; N, 5.67. ${ }^{1} \mathrm{H}$ NMR $\left(500.1 \mathrm{MHz}, \delta, \mathrm{CDCl}_{3}\right): 3.52$ and $3.77\left(6 \mathrm{H}, 2 \mathrm{~s}, 2 \mathrm{CH}_{3}\right), 4.87\left(1 \mathrm{H}, \mathrm{dd},{ }^{2} \mathrm{~J}_{\mathrm{PH}}=21.0 \mathrm{~Hz}\right.$, $\left.{ }^{3} \mathrm{~J}_{\mathrm{HH}}=11.2 \mathrm{~Hz}, \mathrm{P}-\mathrm{CH}-\mathrm{CH}\right), 6.24\left(1 \mathrm{H}, \mathrm{dd},{ }^{3} \mathrm{~J}_{\mathrm{PH}}=8.9 \mathrm{~Hz},{ }^{3} \mathrm{~J}_{\mathrm{HH}}=11.2 \mathrm{~Hz}, \mathrm{P}-\mathrm{CH}-\mathrm{CH}\right), 6.63-8.02(15 \mathrm{H}$, 
m, $\left.\mathrm{H}_{\text {aro }}\right){ }^{13} \mathrm{C}$ NMR $\left(125.8 \mathrm{MHz}, \delta, \mathrm{CDCl}_{3}\right): 47.84\left(\mathrm{~d},{ }^{1} \mathrm{~J}_{\mathrm{CP}}=135.9 \mathrm{~Hz}, \mathrm{P}-\mathrm{CH}\right), 53.28$ and $53.41(\mathrm{~s}$, $\left.2 \mathrm{OCH}_{3}\right), 58.66\left(\mathrm{~d},{ }^{2} \mathrm{~J}_{\mathrm{CP}}=3.9 \mathrm{~Hz}, \mathrm{P}-\mathrm{C}-\mathrm{CH}\right), 109.71$ and $119.62\left(2 \mathrm{C}, \mathrm{C}_{7} \mathrm{H}_{5} \mathrm{~N}_{2}\right), 120.21$ and 120.40 $\left(2 \mathrm{~d},{ }^{3} \mathrm{~J}_{\mathrm{PC}}=4.4 \mathrm{~Hz} \mathrm{C}_{\text {ortho }}\right.$ of $\left.2 \mathrm{C}_{6} \mathrm{H}_{5}\right), 121.60$ and $124.23\left(2 \mathrm{C}, \mathrm{C}_{7} \mathrm{H}_{5} \mathrm{~N}_{2}\right), 125.56$ and $125.64\left(\mathrm{C}_{\text {para }}\right.$ of $\left.2 \mathrm{C}_{6} \mathrm{H}_{5}\right), 127.38\left(1 \mathrm{C}, \mathrm{C}_{7} \mathrm{H}_{5} \mathrm{~N}_{2}\right), 129.38$ and $129.69\left(\mathrm{C}_{\text {meta }}\right.$ of $\left.2 \mathrm{C}_{6} \mathrm{H}_{5}\right), 135.69$ and $140.76(2 \mathrm{C}$, $\left.\mathrm{C}_{7} \mathrm{H}_{5} \mathrm{~N}_{2}\right), 149.36$ and $149.74\left(2 \mathrm{~d},{ }^{2} \mathrm{~J}_{\mathrm{CP}}=9.8 \mathrm{~Hz}, \mathrm{C}_{\text {ipso }}\right.$ of $\left.2 \mathrm{C}_{6} \mathrm{H}_{5}\right), 167.05\left(\mathrm{~d},{ }^{3} \mathrm{~J}_{\mathrm{CP}}=5.9 \mathrm{~Hz}, \mathrm{C}=\mathrm{O}\right)$, $168.23\left(\mathrm{~d},{ }^{2} \mathrm{~J}_{\mathrm{CP}}=19.3 \mathrm{~Hz}, \mathrm{C}=\mathrm{O}\right) .{ }^{31} \mathrm{P}$ NMR $\left(202.4 \mathrm{MHz}, \delta, 11.20\left[\mathrm{~s},(\mathrm{PhO})_{2} \mathrm{P}=\mathrm{O}\right]\right.$.

$\left(2 S^{*}, 3 R^{*}\right)$-Diethyl-2-(indazol -1-yl)-3-(diphenoxyphosphonato)butanedioate (4e). White powder, 95\%, mp=128-130 ${ }^{\circ} \mathrm{C}$. IR $(\mathrm{KBr})\left(v_{\max }, \mathrm{cm}^{-1}\right): 1738$ and $1720(\mathrm{C}=\mathrm{O}), 1272(\mathrm{P}=\mathrm{O})$. Anal. Calcd for $\mathrm{C}_{27} \mathrm{H}_{27} \mathrm{~N}_{2} \mathrm{O}_{7} \mathrm{P}$ (522): C, 62.21; H, 5.11; N, 5.41, Found: C, 62.07; H, 5.17; N, 5.36. ${ }^{1} \mathrm{H}$ $\operatorname{NMR}\left(500.1 \mathrm{MHz}, \delta, \mathrm{CDCl}_{3}\right): 1.14$ and $1.25\left(6 \mathrm{H}, 2 \mathrm{t},{ }^{3} \mathrm{~J}_{\mathrm{HH}}=7.1 \mathrm{~Hz}, 2 \mathrm{CH}_{3}\right), 4.13$ and $4.25(4 \mathrm{H}, \mathrm{m}$, $2 \mathrm{ABX}_{3}$ system, $\left.2 \mathrm{OCH}_{2}\right), 4.59\left(1 \mathrm{H}, \mathrm{dd},{ }^{2} \mathrm{~J}_{\mathrm{PH}}=21.5 \mathrm{~Hz},{ }^{3} \mathrm{~J}_{\mathrm{HH}}=11.1 \mathrm{~Hz}, \mathrm{P}-\mathrm{CH}-\mathrm{CH}\right), 5.67(1 \mathrm{H}, \mathrm{dd}$, $\left.{ }^{3} \mathrm{~J}_{\mathrm{PH}}=8.8 \mathrm{~Hz},{ }^{3} \mathrm{~J}_{\mathrm{HH}}=11.1 \mathrm{~Hz}, \mathrm{P}-\mathrm{CH}-\mathrm{CH}\right), 6.20-7.56\left(15 \mathrm{H}, \mathrm{m}, \mathrm{H}_{\mathrm{aro}}\right) .{ }^{13} \mathrm{C}$ NMR $(125.8 \mathrm{MHz}, \delta$, $\left.\mathrm{CDCl}_{3}\right): 14.13$ and $14.39\left(2 \mathrm{CH}_{3}\right), 45.33\left(\mathrm{~d},{ }^{1} \mathrm{~J}_{\mathrm{CP}}=132.3 \mathrm{~Hz}, \mathrm{P}-\mathrm{CH}\right), 62.50\left(\mathrm{~d},{ }^{2} \mathrm{~J}_{\mathrm{CP}}=3.7 \mathrm{~Hz}, \mathrm{P}-\mathrm{C}-\right.$ $\mathrm{CH}), 62.67$ and $62.94\left(2 \mathrm{OCH}_{2}\right), 109.54$ and $119.78\left(2 \mathrm{C}, \mathrm{C}_{7} \mathrm{H}_{5} \mathrm{~N}_{2}\right), 120.68$ and $120.91\left(2 \mathrm{~d},{ }^{3} \mathrm{~J}_{\mathrm{PC}}=\right.$ $4.3 \mathrm{~Hz} \mathrm{C}_{\text {ortho }}$ of $\left.2 \mathrm{C}_{6} \mathrm{H}_{5}\right), 121.15$ and $124.56\left(2 \mathrm{C}, \mathrm{C}_{7} \mathrm{H}_{5} \mathrm{~N}_{2}\right), 125.61$ and $125.68\left(\mathrm{C}_{\mathrm{para}}\right.$ of $\left.2 \mathrm{C}_{6} \mathrm{H}_{5}\right)$, $127.25\left(1 \mathrm{C}, \mathrm{C}_{7} \mathrm{H}_{5} \mathrm{~N}_{2}\right), \quad 129.56$ and $129.71\left(\mathrm{C}_{\text {meta }}\right.$ of $\left.2 \mathrm{C}_{6} \mathrm{H}_{5}\right), 135.84$ and $141.04\left(2 \mathrm{C}, \mathrm{C}_{7} \mathrm{H}_{5} \mathrm{~N}_{2}\right)$, 149.69 and $150.05\left(2 \mathrm{~d},{ }^{2} \mathrm{~J}_{\mathrm{CP}}=9.6 \mathrm{~Hz}, \mathrm{C}_{\mathrm{ipso}}\right.$ of $\left.2 \mathrm{C}_{6} \mathrm{H}_{5}\right), 166.16\left(\mathrm{~d},{ }^{3} \mathrm{~J}_{\mathrm{CP}}=6.1 \mathrm{~Hz}, \mathrm{C}=\mathrm{O}\right), 167.21(\mathrm{~d}$, $\left.{ }^{2} \mathrm{~J}_{\mathrm{CP}}=19.5 \mathrm{~Hz}, \mathrm{C}=\mathrm{O}\right) .{ }^{31} \mathrm{P}$ NMR $\left(202.4 \mathrm{MHz}, \delta, 10.85\left[\mathrm{~s},(\mathrm{PhO})_{2} \mathrm{P}=\mathrm{O}\right]\right.$.

\section{$\left(2 S^{*}, 3 R^{*}\right)$-Di-tert-butyl -2-(indazole -1-yl)-3-(diphenoxyphosphonato)butanedioate (4f).}

White powder, $91 \%, \mathrm{mp}=112-115^{\circ} \mathrm{C}$. IR $(\mathrm{KBr})\left(v_{\max }, \mathrm{cm}^{-1}\right): 1736$ and $1725(\mathrm{C}=\mathrm{O}), 1283(\mathrm{P}=\mathrm{O})$. Anal. Calcd for $\mathrm{C}_{31} \mathrm{H}_{35} \mathrm{~N}_{2} \mathrm{O}_{7} \mathrm{P}$ (578): C, 64.51; H,5.97; N, 4.86, Found: C, 64.36; H, 6.06; N, 4.84. ${ }^{1} \mathrm{H}$ NMR (500.1 MHz, $\left.\delta, \mathrm{CDCl}_{3}\right): 1.26$ and $1.52\left(18 \mathrm{H}, 2 \mathrm{CMe}_{3}\right), 4.73\left(1 \mathrm{H}, \mathrm{dd},{ }^{2} \mathrm{~J}_{\mathrm{PH}}=22.1 \mathrm{~Hz}\right.$, $\left.{ }^{3} \mathrm{~J}_{\mathrm{HH}}=11.2 \mathrm{~Hz}, \mathrm{P}-\mathrm{CH}-\mathrm{CH}\right), 6.02\left(1 \mathrm{H}, \mathrm{dd},{ }^{3} \mathrm{~J}_{\mathrm{PH}}=8.9 \mathrm{~Hz},{ }^{3} \mathrm{~J}_{\mathrm{HH}}=11.2 \mathrm{~Hz}, \mathrm{P}-\mathrm{CH}-\mathrm{CH}\right), 6.67-8.01(15 \mathrm{H}$, $\left.\mathrm{m}, \mathrm{H}_{\text {aro }}\right) .{ }^{13} \mathrm{C}$ NMR $\left(125.8 \mathrm{MHz}, \delta, \mathrm{CDCl}_{3}\right): 27.62$ and $27.75\left(2 \mathrm{CMe}_{3}\right), 48.46\left(\mathrm{~d},{ }^{1} \mathrm{~J}_{\mathrm{CP}}=134.5 \mathrm{~Hz}\right.$, $\mathrm{P}-\mathrm{CH}), 59.70\left(\mathrm{~d},{ }^{2} \mathrm{~J}_{\mathrm{CP}}=4.0 \mathrm{~Hz}, \mathrm{P}-\mathrm{C}-\mathrm{CH}\right), 83.13$ and $83.37\left(2 \mathrm{CMe}_{3}\right), 109.82$ and $119.58(2 \mathrm{C}$, $\left.\mathrm{C}_{7} \mathrm{H}_{5} \mathrm{~N}_{2}\right), \quad 120.32$ and $120.72\left(2 \mathrm{~d},{ }^{3} \mathrm{~J}_{\mathrm{PC}}=4.7 \mathrm{~Hz} \mathrm{C}_{\text {ortho }}\right.$ of $\left.2 \mathrm{C}_{6} \mathrm{H}_{5}\right), 122.19$ and $124.17(2 \mathrm{C}$, $\left.\mathrm{C}_{7} \mathrm{H}_{5} \mathrm{~N}_{2}\right), 125.20$ and $125.49\left(\mathrm{C}_{\text {para }}\right.$ of $\left.2 \mathrm{C}_{6} \mathrm{H}_{5}\right), 126.88\left(1 \mathrm{C}, \mathrm{C}_{7} \mathrm{H}_{5} \mathrm{~N}_{2}\right), 129.28$ and $129.36\left(\mathrm{C}_{\text {meta }}\right.$ of $\left.2 \mathrm{C}_{6} \mathrm{H}_{5}\right), 135.38$ and $140.72\left(2 \mathrm{C}, \mathrm{C}_{7} \mathrm{H}_{5} \mathrm{~N}_{2}\right), 149.68$ and $149.79\left(2 \mathrm{~d},{ }^{2} \mathrm{~J}_{\mathrm{CP}}=9.5 \mathrm{~Hz}, \mathrm{C}_{\text {ipso }}\right.$ of $\left.2 \mathrm{C}_{6} \mathrm{H}_{5}\right)$, $163.89\left(\mathrm{~d},{ }^{3} \mathrm{~J}_{\mathrm{CP}}=6.5 \mathrm{~Hz}, \mathrm{C}=\mathrm{O}\right), 165.63\left(\mathrm{~d},{ }^{2} \mathrm{~J}_{\mathrm{CP}}=20.3 \mathrm{~Hz}, \mathrm{C}=\mathrm{O}\right) .{ }^{31} \mathrm{P}$ NMR $(202.4 \mathrm{MHz}, \delta, 10.85$ $\left[\mathrm{s},(\mathrm{PhO})_{2} \mathrm{P}=\mathrm{O}\right]$.

\section{Acknowledgements}

We gratefully acknowledge financial support from the Research Council of University of Sistan and Balouchestan. 


\section{References}

1. (a) Yavari, I.; Islami, M. R.; Bijanzadeh, H. R. Tetrahedron 1999, 55, 5547. (b) Yavari, I.; Ramazani, A. Phosphorus, Sulphur, and Silicon 1997, 130, 73. (c) Yavari, I.; AnariAbbasinejad, M.; Hossaini, Z. Org. Biomol. Chem. 2003, 1, 560.

2. Hudson, H. R. In Chemistry of Organophosphorus Compounds, Vol. 1. Primary, Secondary and Tertiary Phosphines, Polyphosphines and Heterocyclic Organophosphorus (III) Compounds, Hantley, F. R., Wiley: New York, 1990, pp 386-472.

3. Engel, R. Synthesis of Carbon-Phosphorus Bond, CRC Press: Boca Raton, FL, 1988.

4. Cadogan, J. I. G. Organophosphorus Reagents in Organic Synthesis, Academic: New York, 1979.

5. Maryano,B. E.; Reitz, A. B. Chem. Rev. 1989, 89, 863.

6. Cherkasov, R. A. ; Pudovik, M. A. Russ. Chem. Rev. 1994, 63, 1019.

7. Arduago, A. J. III ,Stewart, C. A. Chem. Rev. 1994, 94, 1215.

8. Pietrusiewicz, K. M. ; Zablocka, M. Chem. Rev. 1994, 94, 1375.

9. Bestmann, H. J. ; Vostrowsky, O. Topics Curr. Chem. 1983, 109, 86.

10. George, M. V.; Khetan, S. K ; Gupta, R. K. Adv. Heterocyl. Chem. 1976, 19, 354.

11. Burgada, R.; Leroux, Y.; El Khoshnieh, Y. U. Tetrahedron Lett. 1981, 22, 3533.

12. (a) Castelot-Deliencourt, G.; Pannecoucke, X.; Quirion, J-C. Tetrahedron Lett. 2001, 42, 1025. (b) Cohen, R. J.; Fox, D. L.; Eubank, J. F.; Salvatore, R. N. Tetrahedron Lett. 2003, 44, 8617. (c) Amiguse, E. J.; Migaud, M. E.; Tetrahedron Lett. 2004, 45, 1001. (d) Rassukana, Y. V.; Onys'ko, P. P.; Davydova, K. O.; Sinitsa, A. D. Tetrahedron Lett. 2004, 45, 3899. (e) Onys'ko, P. P.; Suvavola, O. A,; Rassukana, Y. V.; Chudakova, T. I.; Sinitsa, A. D. Tetrahedron Lett. 2003, 44, 1855. (f) Moravcova, J.; Heissigerova, H.; Kocalka, P.; Imberty, A.; Sykora, D.; Fris, M. Tetrahedron Lett. 2003, 44, 8797.

13. (a) Lefebver, I. M.; Evans, Jr., S. A. J. Org. Chem. 1997, 62, 7532. (b) Kim S. K.; Hurh, E. Y.; Youn, J. N.; Park, J. I. J. Org. Chem. 1999, 64, 9272. (c) Joly, D. G.; Jacobsen, E. N. J. Am. Chem. Soc. 2004, 126, 4102. (d) Segall, Y.; Quistand, G. B.; Sparks, S. E.; Casida, J. E. Chem. Res. Toxicol. 2003, 16, 350.

14. (a) Corbridge, D. E. C. Phosphorus; Elsevier: Amsterdam, 1990; Chap. 13, pp 879-954; (b) Quin, D. L. A Guide to Organophosphorus Chemistry; John Wiley: New York, 2000. (c) Walker, B. J. Organophosphorus Chemistry, Penguin Education, 1972. (d) Goodman, Gilman, A.; Hardman, J. G.; Limbird, L. E. The Pharmacological Basis of Therapeutics, 10th Edn., McGraw-Hill: New York, 2001. (e) Gallant. J. E.; Pham. P. A. Expert Rev. Antiinfect. Ther. 2003, 1, 415.

15. (a) Karplus, M. J. Am. Chem. Soc. 1963, 88, 2870. (b) Haasnoot, C. A. G.; de Leeuw, F. A. A. M.; Altona, C. Tetrahedron 1980, 36, 2783.

16. Breitmaier, E.; Voelter, W. Carbon-13 NMR Spectroscopy, VCH: New York, $3^{\text {rd }}$ Edn., 1990; p 250. 
17. Moonen, K.; Van Meenen, E.; Verwee, A.; Stevens, C.V. Angew. Chem. Int. Ed. 2005, 44, 7407.

18. Moonen, K.; Laureyn, I.; Stevens, C. V. Chem. Rev. 2004, 104, 6177

19. K. Brel, V. Synthesis 2002, 13, 1829.

20. Kalantari, M.; Islami, M. R.; Hasani, Z.; Saidi, K. Arkivoc 2006, (x), 55.

21. Islami, M. R.; Mollazehi, F.; Sheibani, H. Arkivoc 2005, (xy), 25.

22. Hassani, Z.; Islami, M. R.; Sheibani, H.; Kalantari, M.; Saidi, K. Arkivoc, 2006, (i), 89. 